\title{
Differentiation of nestin-negative human hair follicle outer root sheath cells into neurons in vitro
}

\author{
WEI WU ${ }^{1}$, XIAO-LI WU ${ }^{1}$, YU-QING $\mathrm{JI}^{2}$ and ZHEN GAO ${ }^{1}$ \\ ${ }^{1}$ Department of Plastic and Reconstructive Surgery, Shanghai Ninth People's Hospital, \\ Shanghai Jiao Tong University School of Medicine, Shanghai 200011; ${ }^{2}$ Department of Plastic \\ and Reconstructive Surgery, Shanghai General Hospital, Shanghai 200080, P.R. China
}

Received February 28, 2016; Accepted February 21, 2017

DOI: $10.3892 / \mathrm{mmr} .2017 .6585$

\begin{abstract}
A specialized quiescent population of hair follicle stem cells, residing in the hair follicle outer root sheath cells (ORSCs), has previously demonstrated pluripotency for differentiation into neural stem cells (NSCs). A previous study indicated that nestin-positive hair follicle ORSCs are able to differentiate into neurons. However, little has been reported on the isolation of nestin-negative human ORSCs and whether they can successfully differentiate into neurons in vitro. In the present study, nestin-positive ORSCs were significantly reduced with a prolonged incubation time in vitro. Following 9 days of primary culture, nestin-expressing ORSCs disappeared entirely, and ORSCs remained nestin-negative following 5 days of subculture. Notably, nestin was identified in ORSCs following a three-step process of neuro-induction. In addition, neruodevelopmental markers were detected in the ORSC-derived nestin-positive spherical cell mass, including the induction of the neuronal specific markers growth associated protein- 43 , neurotensin receptor- 3 and p75 neurotrophin receptor, and also the gliocyte markers, glial fibrillary acidic protein and S100. These sphere-forming cells did not express the mature neuron-associated markers neurofilament medium, neuronal nuclei and neuron-specific enolase, which suggested that sphere-forming cells may preferentially differentiate into neural stem cell-like cells as opposed to mature neurons or neurogliocyte. In conclusion, ORSC-driven neural differentiation may be a suitable treatment strategy for neurodegenerative diseases and may possess an important value in regenerative medicine.
\end{abstract}

Correspondence to: Dr Zhen Gao, Department of Plastic and Reconstructive Surgery, Shanghai Ninth People's Hospital, Shanghai Jiao Tong University School of Medicine, 639 Zhizaoju Road, Shanghai 200011, P.R. China

E-mail: gaozhen_nf@163.com

Key words: nestin, human hair follicle outer root sheath cells, neuron, cytothesis, differentiation

\section{Introduction}

It is assumed that most peripheral tissues have at least a limited ability for self-repair however, the central nervous system (CNS) is known to be relatively resistant to regeneration (1). In general, although neural stem cells (NSCs) have the potential to generate a large number of specific neural phenotypes in vitro, including neurons, neurogliocytes and Schwann cells, NSCs do not participate in neuropathy plerosis (2). In addition, a small number of stem cells have been demonstrated to reprogramme to an alternative differential fate to become a neuron (3). The majority of differentiation follows a unidirectional and irreversible route, therefore the development of novel approaches to generate neurons from non-neural lineages are required. This would have important implications for the study of neural development, neurological disease modelling and regenerative medicine.

A specialized quiescent population of outer root sheath cells (ORSCs), residing in the hair follicle, has exhibited pluripotency for differentiation into epithelial-mesenchymal lineage cells (4). A previous study indicated that human ORSCs have the potential for developing non-invasive treatments for skin disorders (5). Due to improved culture techniques, ORSCs can be induced to develop highly differentiated epidermal equivalents (6). Notably, it has been reported that implantation of multipotent nestin-positive hair follicle stem cells promotes the repair of spinal cord and peripheral nerves (7-9). However, little has been reported on the isolation of nestin-negative human ORSCs and whether they can successfully differentiate into nerve cells in vitro. The aim of the present study was to isolate nestin-negative ORSCs from adult healthy volunteers and investigate the neuronal differentiation of these cells.

\section{Materials and methods}

Cell isolation and culture. The present study included a total of 10 individuals (with a mean age of $45.2 \pm 9.8$ years). All participants were clinically free of disease, including hair and/or scalp disease, for $\geq 6$ months, as defined by medical history and physical examination. The subjects were recruited from September 2014 to January 2015 from the Shanghai Ninth People's Hospital, Shanghai Jiao Tong 
University, School of Medicine, Shanghai, China. Human scalp samples were obtained with written informed consent from all participants. The study was approved by the Ethics Committee of the Shanghai Ninth People's Hospital, Shanghai Jiao Tong University, School of Medicine, Shanghai, China. Human scalp samples were soaked in Dulbecco's modified Eagle's medium (DMEM; Gibco; Thermo Fisher Scientific, Inc., Waltham, MA, USA) containing $0.5 \%$ penicillin and $0.5 \%$ streptomycin, for $\leq 6 \mathrm{~h}$. The epidermis and adipose tissue was then peeled away from the dermis, cut into small fragments using scissors and treated with dispase $(12.5 \mathrm{mg} / \mathrm{ml}$; Roche Applied Science, Penzberg, Germany) for $24 \mathrm{~h}$ at room temperature. The suspension was digested with $0.1 \%$ trypsin for $30 \mathrm{~min}$ at $37^{\circ} \mathrm{C}$ and filtered through a cell strainer $(40 \mu \mathrm{m}$ diameter holes). ORSCs were obtained by centrifugation at $1,000 \mathrm{x} \mathrm{g}$ for $5 \mathrm{~min}$ at $4^{\circ} \mathrm{C}$. Subsequently, cells were cultured in medium containing epidermal growth factor $(20 \mathrm{ng} / \mathrm{ml})$, basic fibroblast growth factor (b-FGF; $40 \mathrm{ng} / \mathrm{ml}$ ), and 2\% B27 supplement (Gibco-BRL; Thermo Fisher Scientific, Inc.) in DMEM/F12 (1:1; Gibco-BRL; Thermo Fisher Scientific, Inc.) in $5 \% \mathrm{CO}_{2}$ at $37^{\circ} \mathrm{C}$ for 2 weeks.

Human hair follicle ORSC passage culture and neural differentiation. Following primary culture for 2 weeks, the spheres were dissociated by pipetting, and the cells were subcultured for 5 days. For experiments, cells at the first subculture were used. A three-step process of neuro-induction was performed on isolated sphere-forming cells. First, $2 \times 10^{5}$ sphere-forming cells were cultured in medium containing DMEM-high glucose (HG) medium with $20 \%$ fetal bovine serum (FBS) and $10 \mathrm{ng} / \mathrm{ml}$ b-FGF (R\&D Systems, Inc., Minneapolis, MN, USA) for $24 \mathrm{~h}$. The sphere-forming cells were then washed with phosphate-buffered saline three times and cultured in DMEM-HG medium containing $1 \mathrm{mM} \beta$-mercaptoethanol (Sigma; Merck KGaA, Darmstadt, Germany) and $10 \mathrm{ng} / \mathrm{ml}$ neurotrophin (NT)-3 (R\&D Systems, Inc.) for $48 \mathrm{~h}$. Following this, the sphere-forming cells were cultured in DMEM-HG medium containing $10 \mathrm{ng} / \mathrm{ml} \mathrm{NT-3,10} \mathrm{ng/ml} \mathrm{nerve} \mathrm{growth}$ factor (NGF) and $50 \mathrm{ng} / \mathrm{ml}$ brain-derived neurotrophic factor (BDNF; R\&D Systems, Inc.) for 5 days. The total duration of neural differentiation was 8 days. The primary culture of ORSCs following 2 days (passage 0, day 2; P0D2) and the subculture sphere-forming cells prior neural differentiation (P1D5) acted as control groups.

Immunofluorescence staining. Following dissociation of the sphere-forming cells, the cells were fixed in $4 \%$ paraformaldehyde and rinsed with PBS three times. Non-specific binding was blocked in PBS containing 5\% bovine serum albumin (Sigma) and $0.1 \%$ Triton $\mathrm{X}-100$ at $37^{\circ} \mathrm{C}$ for $1 \mathrm{~h}$. A total of $1 \times 10^{5}$ cells were characterized immunocytochemically using an anti-nestin antibody (catalog no. sc-23927; 1:50; Santa Cruz Biotechnology, Inc., Dallas, TX, USA), anti-cytokeratin (CK) antibody (catalog no. M082101-2; 1:100; Dako; Agilent Technologies, Inc., Santa Clara, CA, USA), anti-CK15 antibody (catalog no. ab52816; 1:100; Abcam, Cambridge, UK), anti-CK18 antibody (catalog no. sc-70917; 1:50; Santa Cruz Biotechnology, Inc.), anti-CK19 antibody (catalog no. MA5-13156; 1:150; NeoMarkers; Thermo Fisher Scientific, Inc.), anti-follistatin (FST) antibody (catalog no. MAB669; 1:100; R\&D Systems, Inc.), anti-growth associated
protein(GAP)-43 antibody (catalog no.sc-33705; 1:50; SantaCruz Biotechnology, Inc.), anti-glial fibrillary acidic protein (GFAP) antibody (catalog no. ab7260; 1:100; Abcam, Cambridge, UK), anti-neuronal nuclei (NeuN) antibody (catalog no. ABN78; 1:50; Chemicon; EMD Millipore, Billerica, MA, USA), anti-neurofilament medium (NF-M) antibody (catalog no. sc-71688; 1:50; Santa Cruz Biotechnology, Inc.), anti-neuron-specific enolase (NSE) antibody (catalog no. MAB324-K; 1:50; Chemicon; EMD Millipore), anti-neurotensin receptor-3 (NTR-3) antibody (catalog no. sc-25055; 1:50; Santa Cruz Biotechnology, Inc.), anti-p75 neurotrophin receptor (catalog no. ab8874; P75 ${ }^{\mathrm{NTR}}$; 1:250; Abcam) and anti-S100 antibody (catalog no. GA50461-2; 1:200; Dako; Agilent Technologies, Inc.). Following washing with PBS containing $0.05 \%$ Tween-20 3 times for $3 \mathrm{~min}$ at room temperature, CK, NSE, NF-M and GAP-43 were visualized using a fluorescein isothiocyanate-conjugated goat anti-mouse IgG (catalog no. F8264; 1:500; Sigma), and CK-19, GFAP, S100 and $\mathrm{p} 75^{\mathrm{NTR}}$ were visualized using rhodamine-conjugated goat anti-rabbit IgG (catalog no. SAB3700846; 1:500; Invitrogen; Thermo Fisher Scientific, Inc.) as secondary antibodies at $37^{\circ} \mathrm{C}$ for $1 \mathrm{~h}$. Nuclei were visualized with DAPI $(0.1 \mathrm{mg} / \mathrm{ml}$; catalog no. D8417; Sigma). Mounting medium (containing 45\% acrylic resin and 55\% xylenes; catalog no. 03989; Sigma) was used for microscopic observation. The immunofluorescent signal was observed under a fluorescence microscope (Leica DM2500; Leica, Wetzlar, Germany).

\section{Results}

Nestin-negative ORSC separation, identification and neural differentiation. Facial skin samples were obtained from 10 adult healthy volunteers, and the epidermis, rich in hair follicles, was peeled off from the dermis following dispase treatment. Subsequently, the epidermis was cut into small fragments, digested with trypsin, and filtered through a $40-\mu \mathrm{m}$-pore cell strainer; ORSCs were obtained by centrifuging the cell suspension. Of the ORSCs dissociated from hair follicles in $48 \mathrm{~h}$ primary cultures, $75 \pm 4 \%$ of them were positive for nestin (red staining) as demonstrated by immunofluorescence staining (Fig. 1A). However, the nestin-positive ORSCs were reduced with prolonged incubation in vitro: Following 9 days of primary culture, nestin expressing ORSCs disappeared entirely in vitro, and ORSCs remained nestin-negative following 5 days of subculture (Fig. 1B). Based on these results, ORSCs were cultured in the presence of neurabasal medium containing DMEM-HG, 20\% FBS and $10 \mathrm{ng} / \mathrm{ml} \mathrm{b}$-FGF. The majority of the cells exhibited colony growth (Fig. 1C), and, except for nestin-negative expression, NTR-3, GAP-43, GFAP, P75 ${ }^{\mathrm{NTR}}$, NSE, NF-M, NeuN, FST, CK15 and CK18 were negatively expression in ORSCs. However, CK19, CK and S100 were only expressed in a small number of ORSCs (Table I). The ORSCs started to form spheres 5 days after the start of the subculture (first-generation) in the presence of neurabasal medium (Fig. 1D), and numerous spheres were connected by a filamentary structure (Fig. 1E).

Immunofluorescent staining of markers for P1 ORSCs following neuro-induction. Eight days after neuroinduction sphere-forming cells were derived from ORSCs, and the single cells were cultured in medium containing DMEM-HG 
Table I. Differentiation marker expressions of passage 1 outer root sheath cells prior to and following neurogenic induction.

A, Neural markers

\begin{tabular}{lccc}
\hline Marker & P0 D2 & P1: Prior to NI & P1: Following NI \\
\hline Nestin & Positive & Negative & Positive \\
NTR-3 & Negative & Negative & Positive \\
GAP-43 & Positive & Negative & Positive \\
P75 & Positive & Negative & Positive \\
GFAP & Positive & Negative & Positive \\
S100 & Rare positive & Rare positive & Positive \\
NSE & Negative & Negative & Negative \\
NF-M & Negative & Negative & Negative \\
NeuN & Negative & Negative & Negative \\
\hline
\end{tabular}

B, Cytokeratin and ORS markers

\begin{tabular}{lccc}
\hline CK & Rare positive & A few positive & Positive \\
CK19 & Positive & A few positive & Positive \\
CK15 & A few positive & Negative & Negative \\
CK18 & Negative & Negative & Negative \\
FST & A few positive & Negative & Negative \\
\hline
\end{tabular}

NI, neurogenic induction; P, passage number; NTR-3, neurotensin receptor-3; GAP-43, growth associated protein- 43 ; P75 ${ }^{\mathrm{NTR}}$, p75 neurotrophin receptor; GFAP, glial fibrillary acidic protein; NSE, neuron-specific enolase; NF-M, neurofilament medium; NeuN, neuronal nuclei; ORS, outer root sheath cells; CK, cytokeratin; FST, follistatin.
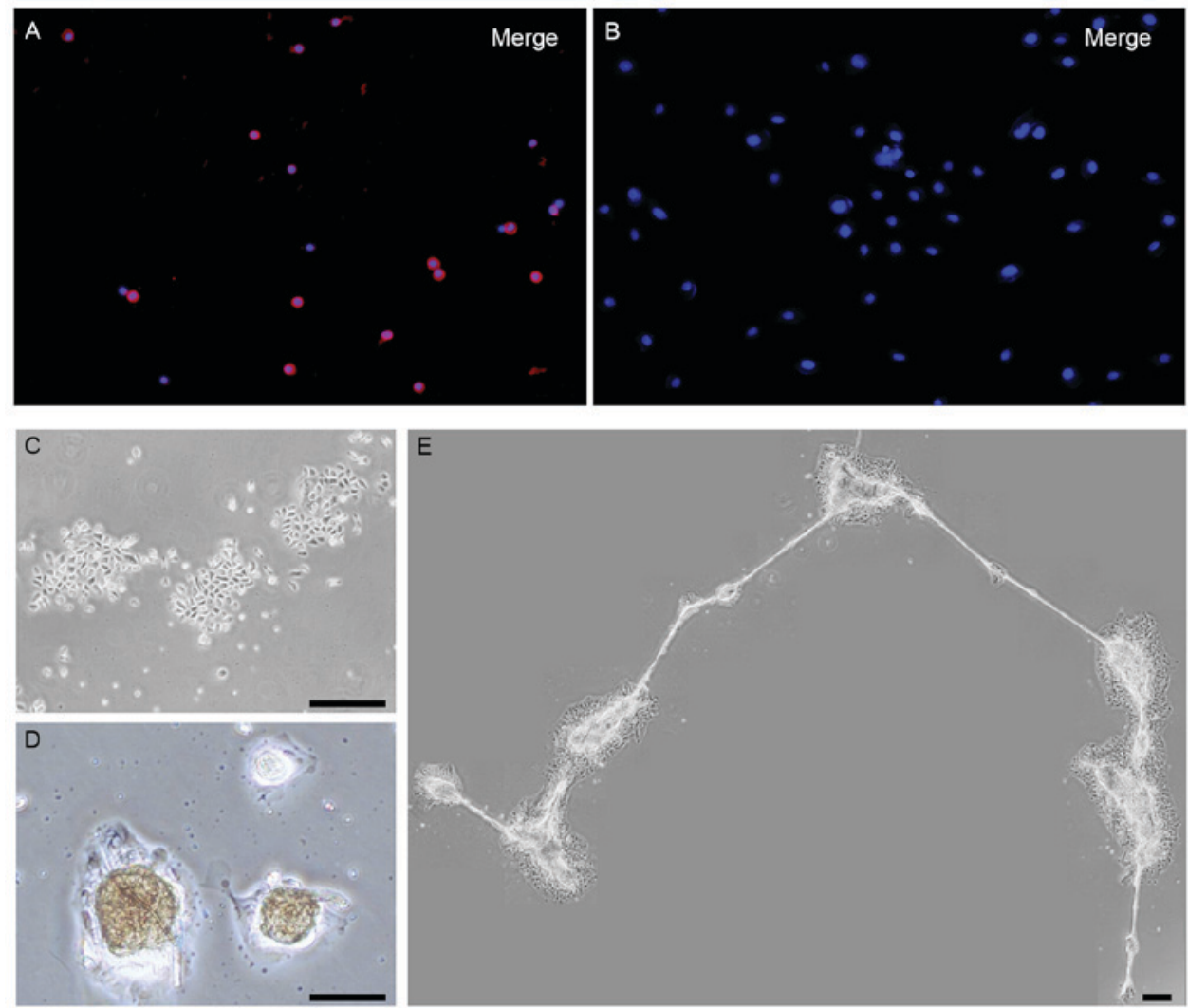

Figure 1. Immunofluorescent staining for nuclei (blue) and nestin (red) following the dissociation of ORSCs from hair follicles after (A) 2 days of primary culture and (B) subculture for 5 days. Magnification=x50. Morphological observation of ORSCs on (C) the 5 day cell passage 1 culture (P1D5) identified (scale bar $=200 \mu \mathrm{m})(\mathrm{D})$ the formation of sphere-forming cells (scale bar=25 $\mu \mathrm{m}$ ), and (E) a filamentary structure formed between the sphere-forming cells (scale bar $=6.25 \mu \mathrm{m})$. ORSC, outer root sheath cells. 

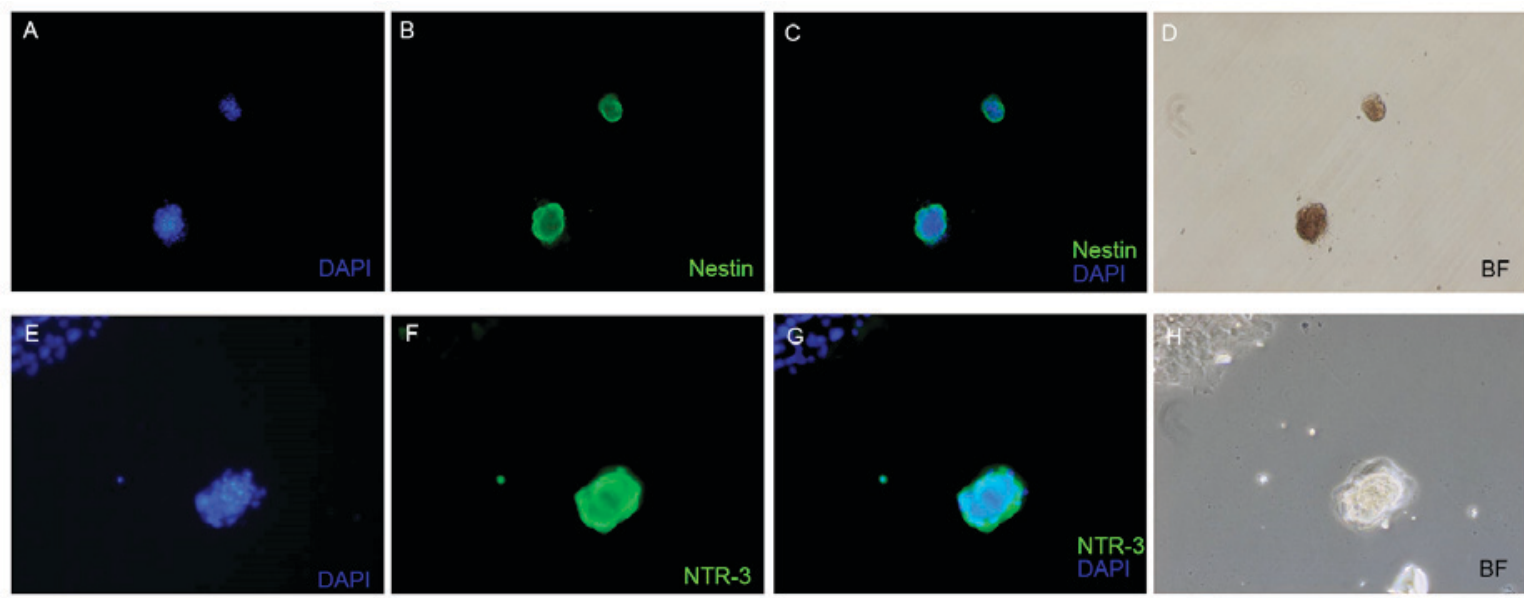

Figure 2. Immunofluorescent staining for (A) nuclei (blue), (B) nestin (green) and (C) merge following neuroinduction, and (D) the corresponding BF image Immunofluorescent staining for (E) nuclei, (F) NTR-3 and (G) merge following neuroinduction, and (H) the corresponding BF image. Magnification, x400. DAPI, 4',6-diamidino-2-phenylindole; NTR-3, neurotensin receptor-3; BF, brightfield.
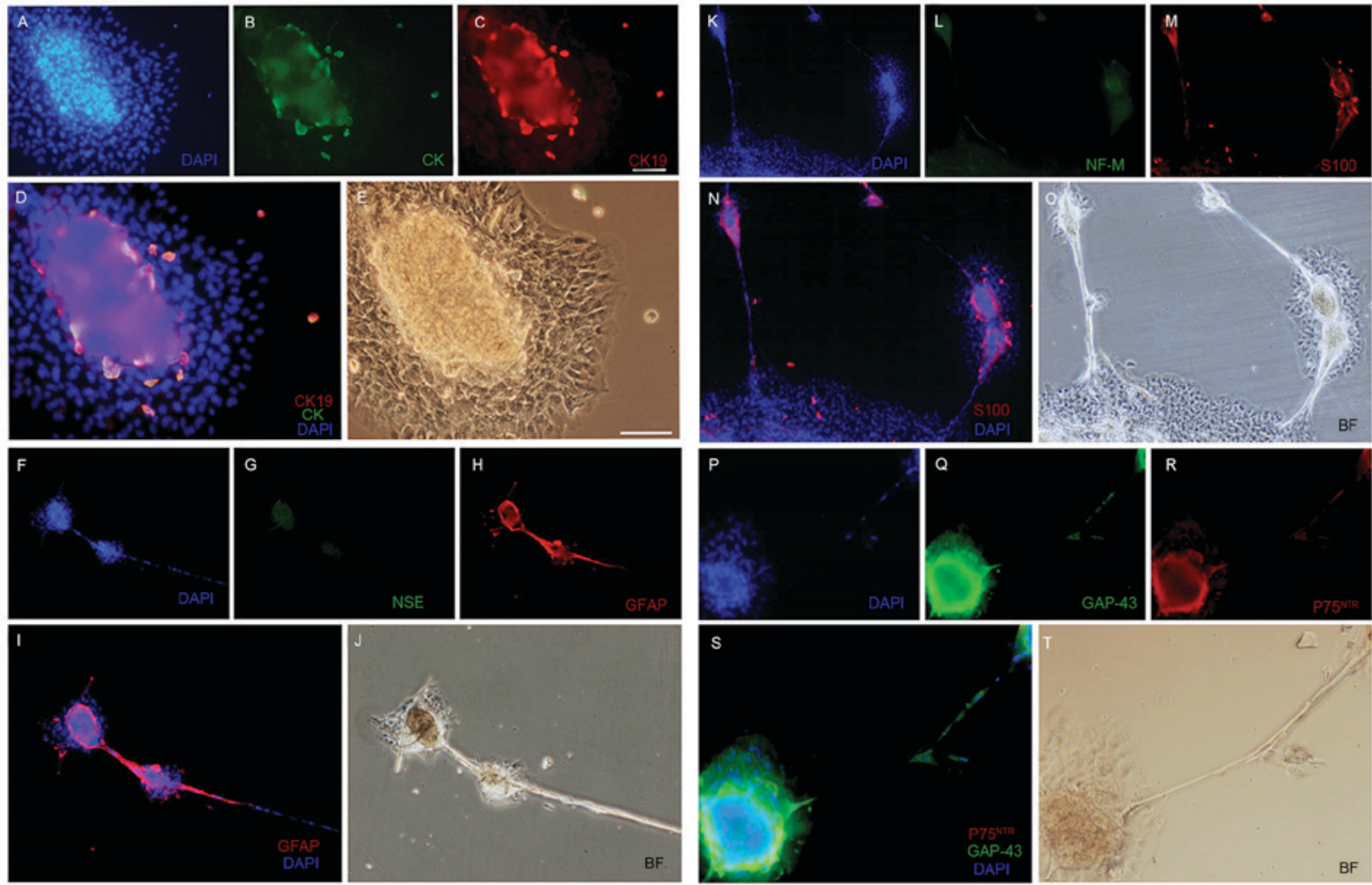

Figure 3. Immunofluorescent staining for (A) nuclei (blue), (B) CK (green) and (C) CK19 (red), (D) merge picture for CK and CK19 and (E) BF control in sphere-forming cells. Immunofluorescent staining for (F) nuclei (blue), (G) NSE (green) and (H) GFAP (red), (I) merge picture for GFAP and (J) BF control in the sphere-forming cells. Immunofluorescent staining for (K) nuclei (blue), (L) NF-M (green) and (M) S100 (red), (N) merge picture for S100 and (O) BF control in the sphere-forming cells. Immunofluorescent staining for (P) nuclei (blue), (Q) GAP-43 (green) and (R) P75 ${ }^{\mathrm{NTR}}$ (red), (S) merge picture for GAP-43 and P75 ${ }^{\mathrm{NTR}}$ and (T) BF control in the sphere-forming cells. Magnification, x400. DAPI, 4',6-diamidino-2-phenylindole; CK, cytokeratin; BF, brightfield; NSE, neuron-specific enolase; GFAP, glial fibrillary acidic protein; NF-M, neurofilament medium; GAP-43, growth associated protein-43; P75 ${ }^{\mathrm{NTR}}$, p75 neurotrophin receptor.

and NT-3 (10 ng/ml), NGF (10 ng/ml) and BDNF (50 ng/ml). Notably, the expression of nestin was identified in ORSCs following a three-step process of neuroinduction (Fig. 2A-D). NTR-3 is present in the brain, particularly in the hippocampus, dentate gyrus and cerebral cortex, indicating that it may have a functional significance in metabolically active brain regions (10). During embryonic brain development, NTR3 mRNA is expressed in all brain areas from E9.5 to E13.5, at which point, dividing cells lining the neural canal and the ventricles, as well as cells already differentiating, express NTR3 (10). The present study observed that NTR-3 was detected in ORSCs following neuroinduction by immunofluorescent staining (Fig. 2E-H). In addition, CK, CK-19 and S100 were expressed within a subset of ORSCs, which were located in an island-shaped structure at the base of the sphere-forming structure (Fig. 3B, C and M, respectively). In addition, NSE 

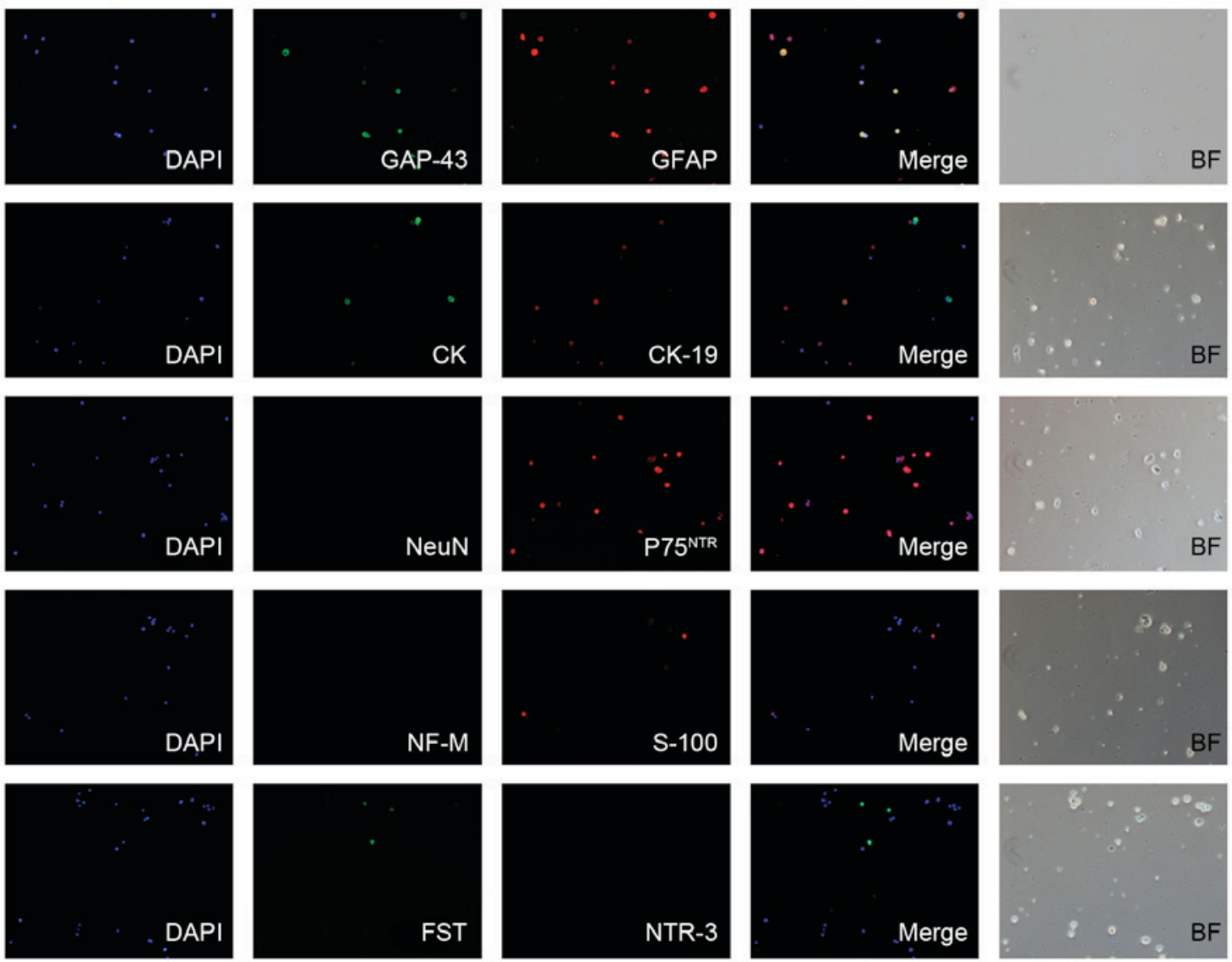

Figure 4. Immunofluorescent staining for neural differentiation markers in the control primary culture of outer root sheath cells at 2 days (P0D2). Magnification, x50. DAPI, 4',6-diamidino-2-phenylindole; GAP-43, growth associated protein-43; GFAP, glial fibrillary acidic protein; CK, cytokeratin; NeuN, neuronal nuclei; P75 ${ }^{\mathrm{NTR}}$, p75 neurotrophin receptor; NF-M, neurofilament medium; FST, follistatin; NTR-3, neurotensin receptor-3; BF, brightfield.

(Fig. 3G), NF-M (Fig. 3L) were not detected in ORSCs following neuroinduction, nor were NeuN, FST, CK15 and CK18 (Table I). Furthermore, GFAP (Fig. 3H and I), GAP-43 (Fig. 3Q and S) and P75 ${ }^{\text {NTR }}$ (Fig. 3R and S) were detected in the majority of the sphere-forming cells. Similar results were obtained in the control group (P0D2), however; NTR-3 was expressed in very low levels (Fig. 4). In P1D5 control group, the immunofluorescent staining revealed that there were low expression levels of CK15, CK19 and S100 in ORSCs, however, no other expression was observed in ORSCs (Table I).

\section{Discussion}

Nestin is an intermediate filament protein that is expressed in a variety of tissues, including those in the CNS and the peripheral nervous system. Nestin is downregulated and replaced by tissue-specific intermediate filament proteins in the progression of tissue differentiation $(11,12)$. Previous studies have demonstrated that nestin is expressed in bulge-area stem cells of the hair follicle, and multipotent nestin-positive hair-follicle bulge stem cells can form neurons $(4,8)$. In non-balding human scalp skin, nestin was detected by immunohistochemical analysis in the epidermis and the upper two-thirds of the hair follicle, however, not in the lower third of the follicle (13). Transfection of nestin-expressing hair-follicle cells into nude-mouse skin, has revealed that they are interconnected by an nestin-driven GFP (ND-GFP)-labeled dermal vascular network, which developed into an extensively branched network, appearing to anastomose with existing vessels in the recipient nude mice. In addition, the nestin-expressing follicle cells contributed to wound repair as well as skin transplant survival $(14,15)$. These results indicated that nestin is present in ORSCs, and that ORSCs are significant in the periodic cycle of hair follicle and wound repair in epidermal tissue. Nervous tissue wound repair and regeneration are the most complex problems in regenerative medicine. However, the use of hair follicle-derived cells to develop nerve regeneration and functional recovery may be a suitable treatment strategy for neurodegenerative diseases and may possess an important value in regenerative medicine.

The present study demonstrated that the isolation of nestin-negative ORSCs derived from the human hair follicle exhibited the neuronal differentiation of these cells. The isolated cells exhibited sphere-forming ability, and nestin was identified in ORSCs following a three-step process of neuroinduction. Similarly, Amoh et al (16) demonstrated that ND-GFP hair-follicle stem cells differentiate into neurons, glia, keratinocytes, smooth muscle cells and melanocytes in vitro, and that ND-GFP-expressing stem cells extensively differentiate into neurons following transplantation to the subcutis of nude mice. In the present study, neurodevelopmental markers were detected in the ORSC-derived nestin-positive spherical cell mass. The neuronal specific markers, GAP-43, NTR-3 and P75 ${ }^{\mathrm{NTR}}$ were induced, and the gliocyte markers GFAP and S100 were detected in these 
sphere-forming cells, however, the mature neuron-associated markers, NF-M, NeuN and NSE were not expressed, suggesting that sphere-forming cells may preferentially differentiate into neural stem cell-like cells and not into the mature neurons or neurogliocytes.

Hair follicle stem cells serve a vital role in neurodegenerative diseases and regenerative medicine. However, to date, no biochemical markers have been identified that serve as indicators of hair follicle stem cell differentiation, therefore, it is difficult to separate and culture these cells. However, it is established that the multifunctional hair follicle stem cells can be isolated from hair follicle ORSCs and are involved in wound repair and neuroinduction $(4,8,17)$. Therefore, hair follicle ORSCs represent a variety of novel approaches to treat neurodegenerative diseases and regenerative medicine. The present study did not verify the value of neural differentiation into sphere-forming cells for therapeutic application, however, the differentiation of nestin-negative human hair follicle ORSCs into neurons in vitro may serve as a theoretical underpinning for neurodegenerative diseases and regenerative medicine.

\section{Acknowledgements}

This study was supported by the National Natural Science Foundation of China (no. 81272109).

\section{References}

1. Zietlow R, Lane EL, Dunnett SB and Rosser AE: Human stem cells for CNS repair. Cell Tissue Res 331: 301-322, 2008.

2. Ninomiya M, Yamashita T, Araki N, Okano H and Sawamoto K: Enhanced neurogenesis in the ischemic striatum following EGF-induced expansion of transit-amplifying cells in the subventricular zone. Neurosci Lett 403: 63-67, 2006.

3. Batista CE, Mariano ED, Marie SK, Teixeira MJ, Morgalla M, Tatagiba M, Li J and Lepski G: Stem cells in neurology-current perspectives. Arq Neuropsiquiatr 72: 457-465, 2014.

4. Kanno H, Kubo A, Yoshizumi T, Mikami T and Maegawa J: Isolation of multipotent nestin-expressing stem cells derived from the epidermis of elderly humans and TAT-VHL peptide-mediated neuronal differentiation of these cells. Int J Mol Sci 14: 9604-9617, 2013
5. Savkovic V, Flämig F, Schneider M, Sülflow K, Loth T, Lohrenz A, Hacker MC, Schulz-Siegmund M and Simon JC: Polycaprolactone fiber meshes provide a 3D environment suitable for cultivation and differentiation of melanocytes from the outer root sheath of hair follicle. J Biomed Mater Res A 104: 26-36, 2016.

6. Limat A and Hunziker T: Use of epidermal equivalents generated from follicular outer root sheath cells in vitro and for autologous grafting of chronic wounds. Cells Tissues Organs 172: 79-85, 2002.

7. Liu F, Uchugonova A, Kimura H, Zhang C, Zhao M, Zhang L, Koenig K, Duong J, Aki R, Saito N, et al: The bulge area is the major hair follicle source of nestin-expressing pluripotent stem cells which can repair the spinal cord compared to the dermal papilla. Cell Cycle 10: 830-839, 2011.

8. Amoh Y, Li L, Campillo R, Kawahara K, Katsuoka K, Penman S and Hoffman RM: Implanted hair follicle stem cells form Schwann cells that support repair of severed peripheral nerves. Proc Natl Acad Sci USA 102: 17734-17738, 2005.

9. Amoh Y, Kanoh M, Niiyama S, Hamada Y, Kawahara K, Sato Y, Hoffman RM and Katsuoka K: Human hair follicle pluripotent stem (hfPS) cells promote regeneration of peripheral-nerve injury: An advantageous alternative to ES and iPS cells. J Cell Biochem 107: 1016-1020, 2009.

10. Mazella J: Sortilin/neurotensin receptor-3: A new tool to investigate neurotensin signaling and cellular trafficking? Cell Signal 13: 1-6, 2001.

11. Michalczyk K and Ziman M: Nestin structure and predicted function in cellular cytoskeletal organisation. Histol Histopathol 20: 665-671, 2005.

12. Yay A, Ozdamar S, Canoz O, Baran M, Tucer B and Sonmez MF: Intermediate filament protein nestin is expressed in developing meninges. Bratisl Lek Listy 115: 718-722, 2014.

13. Wang Y, Zhang Y, Zeng Y, Zheng Y, Fu G, Cui Z and Yang T: Patterns of nestin expression in human skin. Cell Biol Int 30: 144-148, 2006.

14. Amoh Y, Li L, Yang M, Moossa AR, Katsuoka K, Penman S and Hoffman RM: Nascent blood vessels in the skin arise from nestin-expressing hair-follicle cells. Proc Natl Acad Sci USA 101: 13291-13295, 2004.

15. Kang H,Tian L, Son YJ,Zuo Y,Procaccino D, Love F, Hayworth C, Trachtenberg J, Mikesh M, Sutton L, et al: Regulation of the intermediate filament protein nestin at rodent neuromuscular junctions by innervation and activity. J Neurosci 27: 5948-5957, 2007.

16. Amoh Y, Li L, Katsuoka K, Penman S and Hoffman RM: Multipotent nestin-positive, keratin-negative hair-follicle bulge stem cells can form neurons. Proc Natl Acad Sci USA 102: 5530-5534, 2005

17. Yu H, Fang D, Kumar SM, Li L, Nguyen TK, Acs G, Herlyn M and $\mathrm{Xu} \mathrm{X}$ : Isolation of a novel population of multipotent adult stem cells from human hair follicles. Am J Pathol 168: 1879-1888, 2006. 